\title{
Stars and Gas in the Large Interacting Galaxy NGC 6872
}

\author{
Cathy Horellou \\ Onsala Space Observatory, Chalmers University of Technology, SE-439 \\ 92 Onsala, Sweden \\ Bärbel Koribalski \\ Australia Telescope National Facility, CSIRO, P.O. Box 76, Epping \\ 1710, Australia
}

\begin{abstract}
The luminous barred galaxy NGC 6872 is one of the largest spiral galaxies known. Star formation occurs all along the arms, which extend over more than $100 \mathrm{kpc}$. The galaxy experiences tidal perturbations from the nearby companion IC 4970 passing by on a low-inclination, prograde orbit. We have mapped the large-scale distribution and kinematics of the atomic gas (HI) in the NGC 6872/IC 4970 system and carried out $\mathrm{N}$-body simulations with stars and gas. HI is absent from the central region; on the other hand, large gas concentrations are found at the tip of the tidal arms, spatially coincident with the blue stellar clusters and with the peaks of the $\mathrm{H} \alpha$ distribution. We use that remarkable system to investigate the evolution of gas and stars in a close prograde encounter, examine the influence of a dark matter halo on the length of the tidal tails, and test models of collisionally induced star formation.
\end{abstract}

\section{NGC 6872/IC 4970 observed and imagined}

We have used the Australia Telescope Compact Array to map the large-scale distribution and kinematics of the atomic hydrogen (HI) in the interacting galaxies NGC $6872 / \mathrm{IC} 4970$, that were known to contain $\sim 1.8 \times 10^{10} \mathrm{M}_{\odot}$ of HI gas.

In Fig. 1 we show in contours the distribution of the HI-gas, superimposed on a blue-band VLT image. A different contrast was set in the northern and the southern part of the optical image to better display the presence of the blue stellar clusters at the tip of the spiral arms, which coincide with the maxima in the HI-distribution.

Using $\mathrm{N}$-body simulations with stars and gas we were able to reproduce the general morphological and kinematical features. The extent of the tails is very sensitive to the mass and the concentration of the dark matter halo of the main galaxy, modeled here as a rigid body with half the mass of the stellar disk. Fig. 2 shows the results of the simulation 170 Myr after passage of the companion galaxy to perigalacticon. The companion is modeled as a rigid body five times less massive than the primary galaxy. Its position is marked by a star. A complete description of the results will be given in a forthcoming paper. 

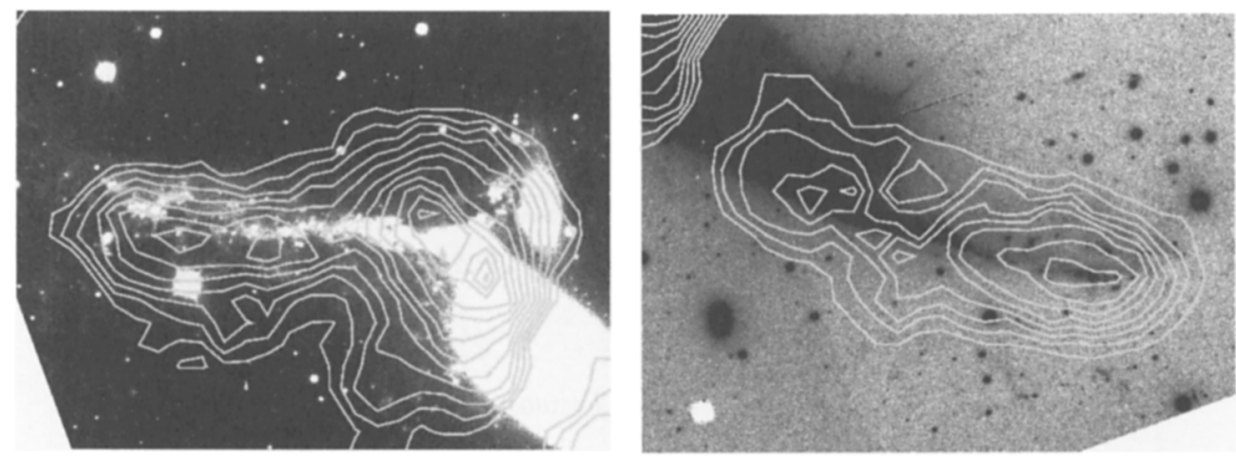

Figure 1. HI $\lambda 21 \mathrm{~cm}$ contour map on a blue-band VLT image. The contour levels range from 0.2 to $1.2 \mathrm{Jy}_{\text {beam }}^{-1} \mathrm{~km} \mathrm{~s}^{-1}$ by steps of 0.1 . The Hi peaks at the tip of the spiral arms coincide with regions of enhanced star formation.

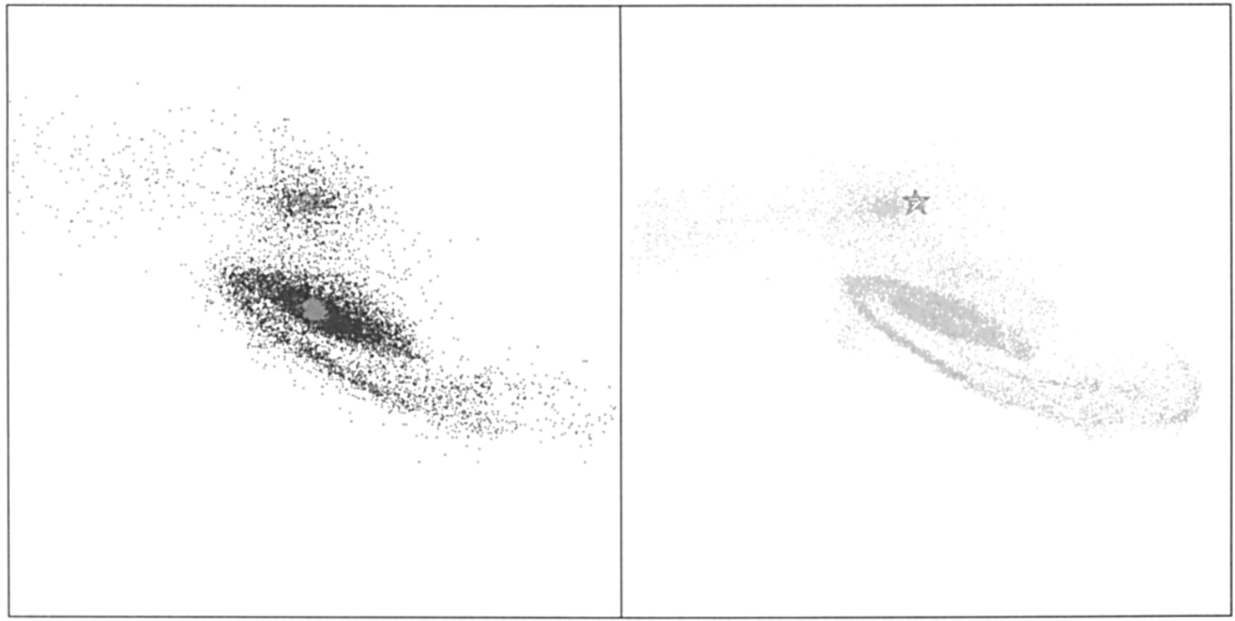

Figure 2. Shapshot of the distribution of the stars (left) and of the gas (right) in our model 170 Myr after passage of the companion to perigalacticon. The position of the companion is indicated by a star. 